\section{(D) Newport.} Brperenes solutions

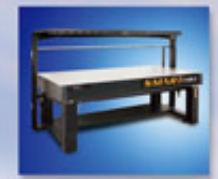

光学隔振平台

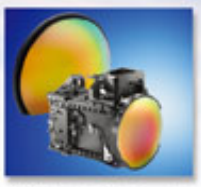

红外及清光学配件

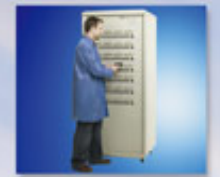

老化则试系统

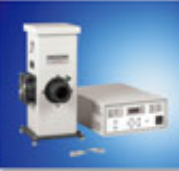

光源
The Brands of Innovation.

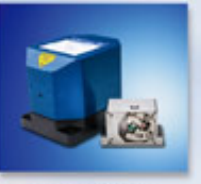

半导体做光器

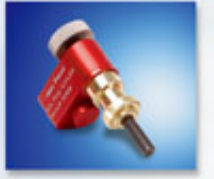

Picomotor ${ }^{2}$ 促动器

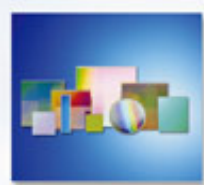

光㨄

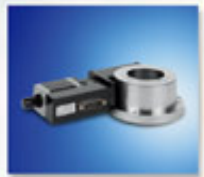

族转台

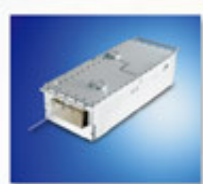

漖光器

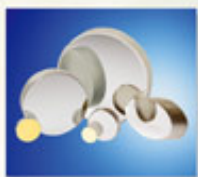

光学镜片

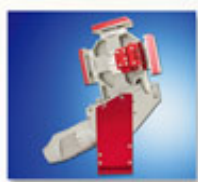

系統集成解决方案
联系我们

Newport中国

电话: +8651081135000

中国区代理商: 科艺仪器有限公司

电话: +864008860019

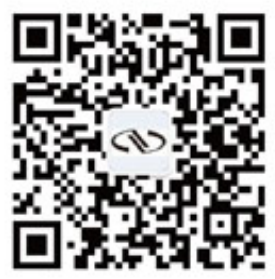

Newport 官方微信 


\title{
SAC-OCDM/TDM system for upgrading the TDM PON
}

\author{
He Chen (陈 荷 $)^{1,2 *}$, Shilin Xiao (肖石林 $)^{1}$, Lilin Yi (义理林 $)^{1}$, \\ Yi Dong (董 毅 $)^{1}$, and Weisheng $\mathrm{Hu}$ (胡卫生) ${ }^{1}$ \\ State Key Laboratory of Advanced Optical Communication Systems and Networks, \\ Shanghai Jiaotong University, Shanghai 200240, China \\ School of Information Science and Engineering, Xinjiang University, Urumqi 830046, China \\ *Corresponding author: gravelhe@gmail.com
}

Received March 7, 2011; accepted April 21, 2011; posted online July 29, 2011

\begin{abstract}
A spectral amplitude coded-optical code division multiplexing time division multiplexing (SACOCDM/TDM) passive optical network (PON) for upgrading the traditional TDM PON is proposed. To the best of our knowledge, our work is the first to report on the use of both spectral and orthogonal code domains, which are transparent to optical network unit (ONU) for hybrid PON, in order to upgrade TDM PON seamlessly. The fewer encoder/decoders and cheaper optical source under the conditions of high bite rate and large ONU accommodation make the system cost-effective. A downstream experiment is demonstrated, and the results demonstrate that the proposed system is feasible.

OCIS codes: $060.2330,060.4230,060.4254$.

doi: 10.3788/COL201109.100606.
\end{abstract}

The time division multiplexing (TDM) technique is used in the Ethernet passive optical network (EPON) and Gigabit passive optical network (GPON), which is currently used commercially and performs optimally. Nextgeneration broadband access networks are a new topic. Therefore, whether there is a need to upgrade directly the bit rates, of EPON and GPON or to introduce new techniques in passive optical network $(\mathrm{PON})$ remains to be elucidated.

Several hybrid PON systems have been proposed to upgrade the traditional TDM PON, such as wavelength division multiplexing (WDM)/TDM PON, optical code division multiplexing (OCDM)/TDM PON, and OCDM/WDM/TDM PON ${ }^{[1-4]}$. These systems provide more optical network units (ONUs) and a larger traffic capacity for PON. However, they present more challenges to the ONU because every ONU must own two-dimentional (2D) or three-dimentional (3D) address information, which will be expensive for subscribers.

In Ref. [5], we have proposed a hybrid wavelength division multiple access (WDMA)/OCDM system which is capable of encoding multiple wavelength channels by employing one encoder and one corresponding optical code. It makes the OCDM transparent to the ONU and so we can upgrade easily the existing WDM PON without redesigning the optical line terminal (OLT) and ONU. Then a full duplex 10G-TDM-OCDM-PON system using only a pair of encoder/decoder was proposed ${ }^{[6]}$. It arranges the multi-port optical encoder/decoder at the remote node $(\mathrm{RN})$ and decreases substantially the number of encoders/decoders. However, the chip rate is 200 Gchip/s for a 16-chip-code, so it presents a challenge to the encoder/decoder and optical source.

In this letter, we propose a novel spectral amplitude coded (SAC)-OCDM/TDM PON. The SAC-OCDM system is based on coding the signal in the spectral domain; the coding is independent of the signal bit rate and is transparent to the ONU. Therefore, the proposed system takes full advantages of the spectral domain, orthogonal code domain, and time domain, and it can be equipped with lower cost devices. Meanwhile, the traditional ONU and OLT in TDM PON do not need to be redesigned in the hybrid PON.

The SAC-OCDM system is considered as one achievement in the optical domain of the CDMA technique ${ }^{[7-9]}$. The code chips are described by spectral slices, where 0 or 1 corresponds to the on or off mode of the spectral slices, respectively. It is based on coding the signal in the optical spectral domain, so the spread spectral gain is independent of the bit and chip rates.

The coding/decoding techniques in SAC-OCDM almost have an identical principle of selecting the spectral chip automatically. When optical pulses arrive, their carrier wave will be split and their spectra will be selected automatically regardless of the time the pulse arrives; the spectral slices are then coupled together. Therefore, we neither need to know the time the optical pulse goes into the optical encoder nor the bit rate of the signals. When the optical signal arrives at the receiver end, the decoder runs autocorrelation, extracts the destined signal, and excludes the interference signals. The optical coding/decoding scheme for SAC-OCDM is independent of the bit rate of the signal from all ONUs and does not change the pulse shape in the time domain if the optical source is a non-coherent broadband source. In comparison, the traditional CDMA in the electric field does not have such property. The new SAC-OCDM/TDM PON is proposed by taking advantage of such unique feature, and its architecture is shown in Fig. 1.

In the proposed system, the hybrid PON is considered to have two layers. The first layer is the traditional TDM PON, and the second layer is based on the SAC-OCDM technique which makes the coding/decoding transparent to the ONU of TDM-PON. In downstream, the data with a high bit rate modulate the amplified spontaneous emission (ASE) source light and then are directed to the encoder. The data after coding can be coupled together with the other $M-1$ data streams in the same or partly overlapped time slot. At the ONU side, the data streams are first selected by the $M$ correspond- 
ing decoders and then are received by $N \times M$ ONUs. The circular is used for double direction transmission. In the upstream, upstream TDM data modulate the light-emitting diode (LED) light individually, and after being coupled together, they are each coded by $M$ tunable encoders/decoders. The data in the same or partly overtime slot are distinguished using different optical orthogonal codes. Then $N \times M$ channel signals are coupled to the single-mode fiber (SMF). At the OLT, the data streams are split into $M$ paths, and the decoder will auto-correlate the data with the destined code and cross-correlate the other undestined data.

In the proposed hybrid PON, we only need $M$ pairs of encoders/decoders to upgrade $N$ users TDM PON to $N \times M$ users hybrid PON, where RN consists of $N+1$ splitters used in traditional TDM PON and $M$ encoders/decoders, and the OLT consists of $N$ ASE sources, $M$ encoders/decoders, and a splitter. Therefore, the upgrading scheme is cost-effective. Based on this two-layer configuration, the coding is independent of the ONUs and transparent to the signal bit rate and signal pattern. As a result, the optical source and optical encoder/decoder will face fewer challenges under the condition of high bit rate or large ONU accommodation, and the network management will be more simple and convenient.

We use a tunable encoder/decoder based on polarization modulation for the SAC-OCDM system, which is employed to achieve a high blocking ratio and lower interference noise ${ }^{[10]}$. The encoder/decoder is used for the hybrid PON. As shown in Fig. 2, when the broadband optical lights from the input port are transmitted into liquid crystal (LC) grating through a collimator, different wavelengths are separated in space (as shown in Fig. 2) and then are incident on different LC cells. Every cell conditioned independently by the electrode will modulate the polarization state of the individual wavelength, which will be directed to the output port or the loss port based on the voltage applied on each cell. Therefore, the spectral component of the output port can be tuned flexibly by the electrode voltage, which can be used for spectral amplitude coding in the OCDM system.

The high blocking ratio of the proposed encoder/decoder can be explained as follows. As shown in Fig. 2, the lights separated in space by LC grating are p-polarized after a p-polarizer. We define the wavelengths to the output port as on-wavelength and those to the loss port as off-wavelength. When we tune the electrode voltage of the LC cells corresponding to the on-wavelength to $4 \mathrm{~V}$, the polarization state of the

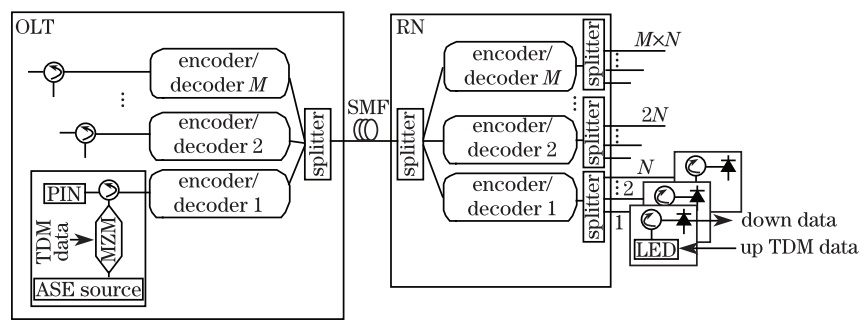

Fig. 1. Architecture of the SAC-OCDM/TDM PON. MZM: Mach-zehnder modulator.

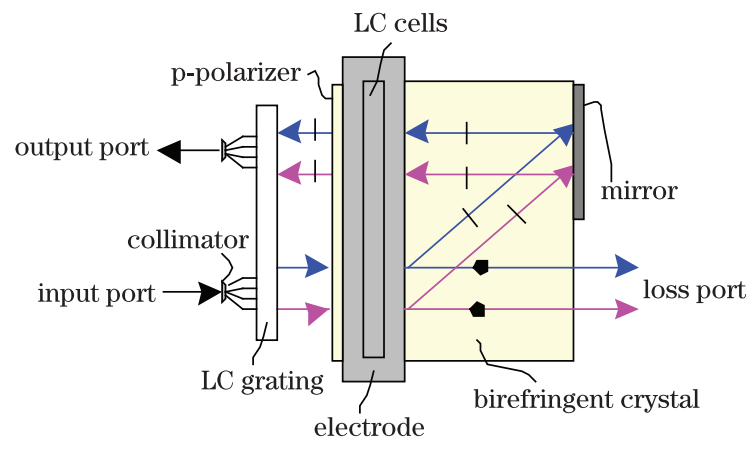

Fig. 2. Optical path of the encoder/decoder.

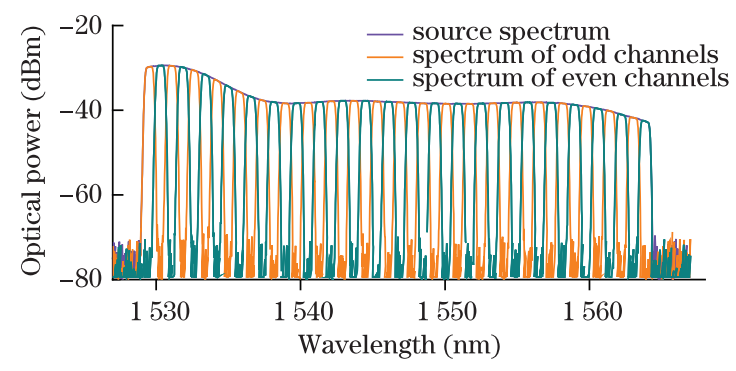

Fig. 3. Block ratio of the LC encoder/decoder.

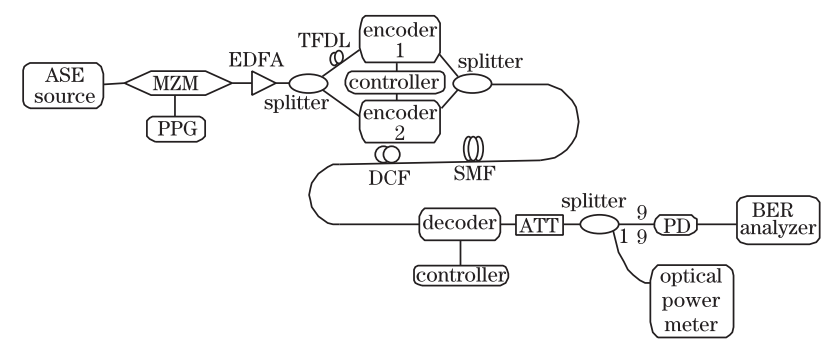

Fig. 4. Experimental setup for the SAC-OCDM/TDM PON.

on-wavelength will not change and still be p-polarized (vertical bar "l" shown in Fig. 2). Then it is guided to the up-path and directed to the output port through a mirror and the LC cells. And only $0.01 \%$ light is lost and leaked to the loss port. For achieving off-wavelength at the loss port, we can tune its corresponding LC cells' voltage to zero. Then $99.9 \%$ light becomes s-polarized (solid dot "•" shown in Fig. 2) and is directed to the loss port, whereas the other $0.1 \%$ light becomes p-polarized and is guided to the up-path. For the up-path p-polarized light, only $0.1 \%$ light is still p-polarized after the LC cells at 0 driving voltage and is directed to the output port, whereas the other $99.9 \%$ light becomes s-polarized and is blocked by the p-polarizer. Therefore, only $0.01 \%$ light of the off-wavelength is leaked to the output port, corresponding to a $40 \mathrm{~dB}$ blocking ratio. Figure 3 shows the blocking performance of the proposed encoder/decoder. The unwanted spectra are blocked to lower than -70 $\mathrm{dBm}$ from the original optical source power of about -30 $\mathrm{dBm}$.

A tunable encoder/decoder with a high blocking ratio for SAC-OCDM can be exploited based on the above principle. The coding/decoding scheme is achieved by controlling the voltage of the corresponding LC cells to be 4 or $0 \mathrm{~V}$ based on " 1 " or " 0 " code in code chips, respectively. 


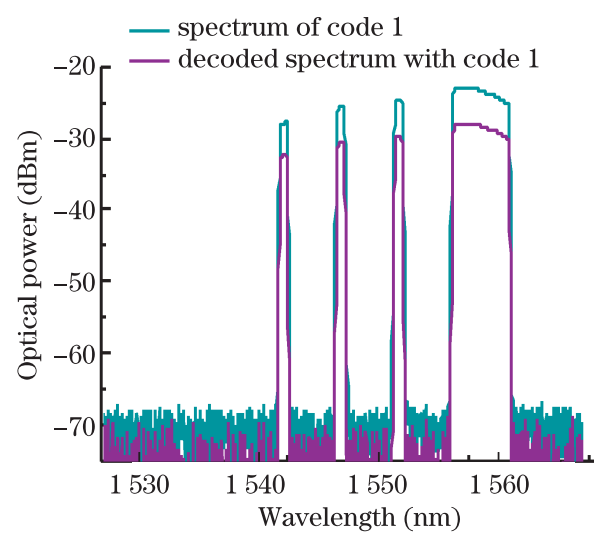

Fig. 5. Spectral slice of code 1.

In the experiment for the proposed SAC-OCDM/TDM PON, we choose the enhanced double weight code as the code address $^{[11]}$. The code length is 45 , and the code weight is 9 . The spectral slices are spaced by $0.8 \mathrm{~nm}$ from 1528.77 to $1564.68 \mathrm{~nm}$. The schematic of the experimental setup is shown in Fig. 4.

In the experiment, the ASE noise from an erbiumdoped fiber amplifier (EDFA) is used as a broadband source in C-band and is modulated by a Mach-Zehnder modulator (MZM) with a $2.5 \mathrm{~Gb} / \mathrm{s}$ non return-to-zero (NRZ) pseudo-random binary sequence (PRBS) data $\left(2^{31}-1\right)$ from a pulse pattern generator (PPG). After being amplified by another EDFA, the signal is divided into two paths by an optical splitter (50:50) and then is coded by encoder 1 and encoder 2, respectively. A tunable fiber delay line (TFDL) is employed on the upchannel for asynchronous transmission of the two channels. At the receiver side, the signal is decoded and then detected by a photodiode (PD). Controllers are used to tune the codes utilized in the encoder and decoder. In the experimental set, one personal computer is applied to control the encoders and decoders. For compensating the strong dispersion of the broadband source, a $170 \mathrm{ps} / \mathrm{nm}$ dispersion compensation fiber (DCF) is used after the 12.9-km SMF. An attenuator (ATT) is employed to vary the received optical power during the measurement of bit error rate (BER). An optical splitter is assigned after the ATT for dividing $99 \%$ of the received power to the $\mathrm{PD}$ and $1 \%$ power to the optical power meter.

First, we tune the code address of encoder 1 and the decoder to code 1: 000000000000000010000010000010 000011111100000 by the controller. Then we make encoder 2 inactive. Figure 5 shows the spectral component of code 1 and the decoded spectrum when the decoder matches encoder 1 . The high block ratio of unwanted spectral slices is promising for a large number of users. The total loss of the proposed encoder/decoder is about $7 \mathrm{~dB}$, and every encoder or decoder may introduce a 3.5-dB insertion loss. The coding performance is then measured. As shown in Fig. 6, the BERs and eye diagrams of back-to-back (BTB) transmission with coding are slightly degraded compared with those without coding. This is because part of the optical spectrum is lost after coding, and the encoder and the decoder may not be perfectly matched. However, when the transmission range is $13 \mathrm{~km}$, and no coding is employed, the dispersion noise is dominant, and the eye closes completely even at
$0 \mathrm{dBm}$ received power as shown in Fig. 6. When the code used in the encoder and the decoder matches, the eye opens again because the dispersion is reduced after spectral amplitude coding.

The OCDM technique is employed for the proposed hybrid PON, so the eye diagram of unmatched code addresses is supposed to be closed. We tune the code address of the encoder to code 2: 00000000000100000100 0001000001111110000000000 , but we maintain the decoder's code address as code 1 . The cross-correlation between code 1 and code 2 will exist in the decoded spectrum (shown in Fig. 7), and it is the interference noise, so the eye diagram of BTB transmission is not fully closed. Clearly, another three spectrum slices (about $-60 \mathrm{dBm})$ appear in the decoded spectrum. They are the inherent interference from the adjacent channels in the encoder, but they are much less compared with the original spectrum so they can be ignored. After $13-\mathrm{km}$ fiber transmission, the eye diagram becomes much worse, so the signal cannot be recovered from unmatched encoders/decoders.

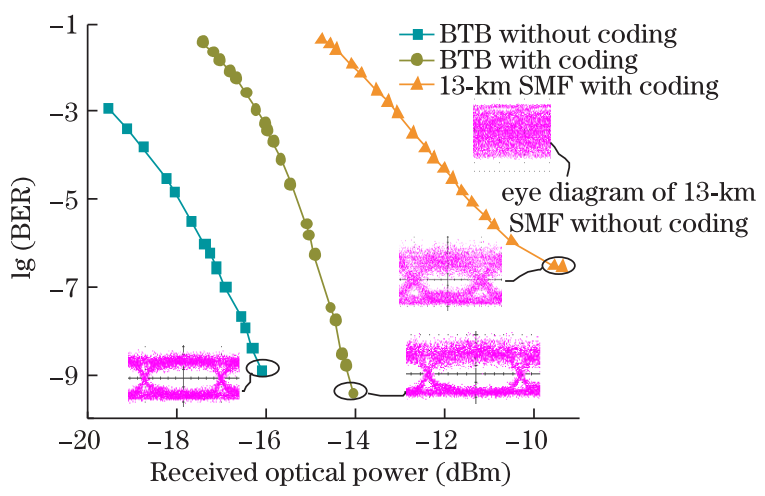

Fig. 6. BER and eye diagram of a single channel.

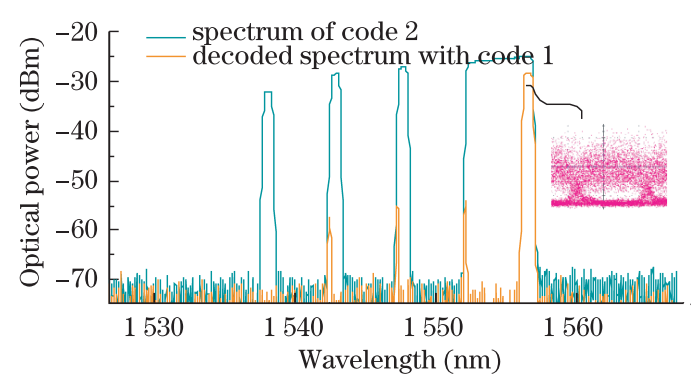

Fig. 7. Spectral slice and eye diagram of unmatched decoding.

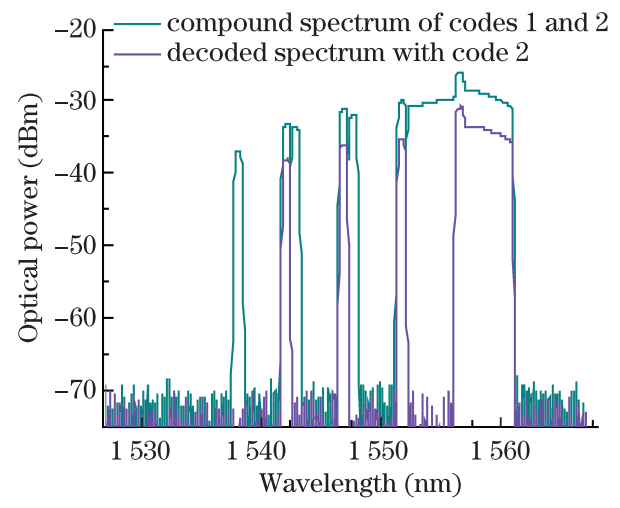

Fig. 8. Spectra of double channels. 


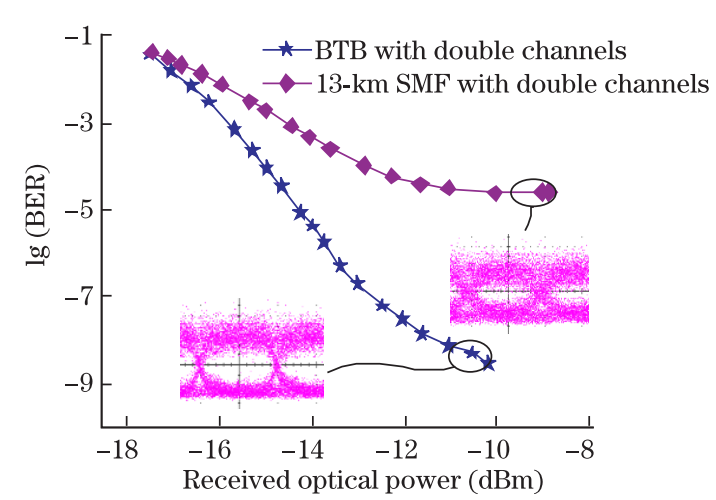

Fig. 9. BER and eye diagram of the double channel.

Then we make both encoder 1 and encoder 2 active, and their codes are set to 0000000000000000100000100 00010000011111100000 for code 1 and 0000000000 01000001000001000001111110000000000 for code 2 . The decoder is tuned to code 1 . The compound spectrum of double channels and the decoded spectrum is shown in Fig. 8. The cross-correlation between code 1 and code 2 embodies a 3 -dB larger power in a compound spectrum and does not disappear in the decoded spectrum as interference noise.

In the double channel experiment, code 1 is the destined receiver. Figure 9 shows the BER performance and eye diagram with a presentation of the interference of code 2. The signal-to-noise ratio is about $3 \mathrm{~dB}$ lower than that in single channel performance as shown in Fig. 6. Therefore, decreasing the multi-user interference in the SAC-OCDM/TDM system is very important.

In conclusion, we propose a hybrid PON which combines the SAC-OCDM and TDM techniques. By utilizing the transparence of optical coding/decoding to the ONUs and the independence of spread spectral gain from the data bit rate in SAC-OCDM technology, the operating TDM PON can be upgraded cost-effectively and seamlessly. Then we exploit a tunable encoder/decoder for the aforementioned hybrid PON. A downstream experiment is simulated, and the result verifies that the proposed SAC-OCDM/TDM PON is feasible.

This work was supported by the National Natural Science Foundation of China (Nos. 60972032 and 61007041) and the National "863" Program of China (Nos. 2006AA01Z251 and 2007AA01Z271).

\section{References}

1. P. J. Urban, B. Huiszoon, R. Roy, M. M. de Laat, F. M. Huijskens, E. J. Klein, G. D. Khoe, A. M. J. Koonen, and H. de Waardt, IEEE/OSA J. Opt. Commun. Netw. 1, A143 (2009).

2. Y. Qian, M. Zhang, D. Liu, L. Deng, and K. Yang, Chin. Opt. Lett. 8, 899 (2010).

3. S. Yoshima, N. Nakagawa, N. Kataoka, N. Suzuki, M. Noda, M. Nogami, J. Nakagawa, and K. I. Kitayama, J. Lightwave Technol. 28, 365 (2010).

4. T. Pfeiffer, J. Kissing, J. P. Elbers, B. Deppisch, M. Witte, H. Schmuck, and E. Voges, J. Lightwave Technol. 18, 1928 (2000).

5. H. Chen, S. Xiao, L. Yi, Y. Dong, and W. Hu, Chin. Opt. Lett. 8, 745 (2010).

6. S. Yoshima, Y. Tanaka, N. Kataoka, N. Wada, and J. Nakagawa, in Proceedings of the European Conference on Optical Communication Tu.3.B.6 (2010).

7. M. Kavehrad and D. Zaccarin, J. Lightwave Technol. 13, 534 (1995).

8. L. Adam, E. S. Simova, and M. Kavehrad, Proc. SPIE 2614, 122 (1995).

9. T. Pfeiffer, B. Deppisch, M. Kaiser, and R. Heidemann, Electron. Lett. 33, 2141 (1997).

10. H. Chen, S. Xiao, L. Yi, Z. Zhou, M. Zhu, J. Shi, Y. Dong, and W. Hu, IEEE Photon. Technol. Lett. (to be published).

11. A. Z. G. Zahid, F. N. Hasoon, and S. Shaari, in Proceedings of International Conference on Future Computer and Communication 658 (2009). 